\title{
INTEGRAL REPRESENTATIONS OF POSITIVE DEFINITE FUNCTIONS. II
}

\author{
BY \\ A. DEVINATZ( $\left.{ }^{1}\right)$
}

1. Introduction. This paper may be considered as a continuation and extension of a previous paper of ours [1], which we shall refer to as IRI. Here, we partially generalize the main theorem of IRI (Theorem 5) to a theorem about the integral representations of positive definite functions whose domains are certain convex sets in a Euclidean space. We shall be more precise about these convex sets a little later. Since many of the results and methods of IRI are used in this paper, it is hoped that the reader will have some acquaintance with the first paper.

One of the theorems which we prove in this paper contains as a special case a theorem of M. Krein [1, p. 17, Theorem 1]. Further, we also obtain as a special case the correct statement of an incorrect theorem stated by $\mathrm{M}$. Livshitz [1, p. 6, Theorem 3]. We shall say more about this later.

We wish to say a few words about our use of the terminology positive definite function. Let $E_{m}$ and $E_{n}$ be the Euclidean spaces of dimension $m$ and $n$ respectively. Let $Q$ be a convex set in $E_{m+n}\left(=E_{m} \times E_{n}\right)$ which contains the origin and such that if $(x, y) \in Q$, for $x \in E_{m}, y \in E_{n}$, then $(x,-y) \in Q$. Further let $Q / 2$ be the set of all $(x, y)$ in $Q$ such that $(2 x, 2 y) \in Q$ and $f(x, y)$ a complexvalued function defined on $Q$. We say that $f$ is a positive definite function if for every finite set of points $\left\{\left(x_{1}, y_{1}\right), \cdots,\left(x_{n}, y_{n}\right)\right\} \subseteq Q / 2$ and every finite set of complex numbers $\left\{\xi_{1}, \cdots, \xi_{n}\right\}$ we have

$$
\sum_{i=1}^{n} \sum_{j=1}^{n} \xi_{i} \bar{\xi}_{j} f\left(x_{i}+x_{i}, y_{j}-y_{i}\right) \geqq 0 .
$$

The terminology positive definite function has been used in the literature for many different things. In particular this title has been given to continuous functions defined on the real axis for which $\sum_{i, j=1}^{n} \xi_{i} \bar{\xi}_{j} f\left(y_{j}-y_{i}\right) \geqq 0$. These functions have also been called functions of positive type. Continuous functions defined on the half real axis for which $\sum_{i, j=1}^{n} \xi_{i} \bar{\xi}_{j} f\left(x_{i}+x_{j}\right) \geqq 0$ have been called exponentially convex functions. Actually, any function of positive type gives rise to a group of unitary operators acting on the reproducing kernel space corresponding to it, while any exponentially convex function gives rise to a semi-group of positive semi-definite self-adjoint operators. From this point

Presented to the Society, April 25, 1952 under the title On positive definite functions; received by the editors July 24, 1953.

(1) Part of this paper was written while the author was at the Illinois Institute of Technology. The major portion was completed while the author was a National Science Foundation Fellow at the Institute for Advanced Study. 
of view it might be really natural to call these latter type functions the positive definite functions. Or better still, in naming them it might be natural to indicate the character of the operators which arise from these functions. At any rate we do not wish to settle any questions of terminology here, and shall leave the ultimate decision to history.

The first part of this paper is concerned with a brief review of some topics which are needed later on. The second part of this paper is concerned with a slight generalization of Theorem 5 of IRI which is needed for the later work. The techniques used in this second part are essentially the same as those used in IRI and we have given a discussion of only those points which we felt might cause the reader some trouble. The third part is concerned with positive definite functions on convex sets. The last part of the paper concerns itself with a problem of moments.

2. Preliminaries. For the convenience of our readers we shall recall briefly some of the essential features of the theory which we shall use in this investigation. Let $E$ be a set (not necessarily having any structure) and $K(x, y)$ a complex-valued function defined on the Cartesian product $E \times E$. The function $K(x, y)$ is called a reproducing $\operatorname{kernel}\left({ }^{2}\right)(\mathrm{r} . \mathrm{k}$.) if there exists a Hilbert space $\mathcal{F}$, of functions defined on $E$, such that $K(x, y) \in \mathcal{F}$ when considered as a function of $x$ for every $y$ in $E$ and for every $f(x) \in \mathcal{F}$ and every $y \in E, f(y)$ $=(f(x), K(x, y)) . \mathcal{F}$ is called a reproducing kernel space (r. k. s.). The function $K(x, y)$ is said to be a positive matrix $\left({ }^{2}\right)$ (p. m.) if for every finite set of complex numbers $\left\{\xi_{1}, \cdots, \xi_{n}\right\}$ and points $\left\{y_{1}, \cdots, y_{n}\right\} \subseteq E$, $\sum_{i, j=1}^{n} \xi_{i} \bar{\xi}_{j} K\left(y_{j}, y_{i}\right) \geqq 0$. The following theorem gives the connection between a r. k. and a p. m.

Theorem A(2). (E. H. Moore-N. Aronszajn). A necessary and sufficient condition that $K(x, y)$ be a r. $k$. is that it be a p. $m$.

For a given p. m. $K(x, y)$, the corresponding space $\mathcal{F}$ is the completion of the set $\mathcal{F}^{\prime}$ of functions of the form

$$
g(x)=\sum_{i=1}^{n} \xi_{i} K\left(x, y_{i}\right),
$$

with norm given by

$$
\|g\|^{2}=\sum_{i, j=1}^{n} \xi_{i} \bar{\xi}_{j} K\left(y_{j}, y_{i}\right) .
$$

As in IRI, $\mathcal{F}$ will always denote the r. $\mathrm{k}$. s. of the $\mathrm{p} . \mathrm{m}$. which we are discussing at a given time and $\mathcal{F}^{\prime}$ will always denote the space of functions given above for that p. m.

Let now $T$ denote an operator from $\mathcal{F}$ to itself, $\mathcal{D}_{T}$ its domain, and $M(x, y)$

(2) N. Aronszajn [1; 2]. 
$\in \mathcal{F}$ for every $y \in E . T$ is said to correspond to the kernel $M(x, y)$, in symbols $T \sim M(x, y)$, if for every $f \in \mathcal{D}_{T}, T f(y)=(f(x), M(x, y)) . T$ is said to correspond to $M(x, y)$ in the maximal sense, in symbols $T \cong M(x, y)$, if $\mathcal{D}_{T}$ consists of all the elements $f$ in $\mathcal{F}$ such that $(f(x), M(x, y))$ is again an element of $\mathcal{F}$, when considered as a function of $y$ and for every $f \in \mathcal{D}_{T}, T f(y)=(f(x), M(x, y))$.

Following Aronszajn we write $M_{1}(x, y) \ll M_{2}(x, y)$ or $M_{2}(x, y)$ $\gg M_{1}(x, y)$ if $M_{2}(x, y)-M_{1}(x, y)$ is a p. $\mathrm{m}$. We have the following theorem.

TheOREM $\mathrm{B}\left({ }^{3}\right)$. Let $M(x, y)$ be a Hermitian symmetric function (i.e. $M(x, y)$ $=\bar{M}(y, x))$ defined over $E \times E$ which belongs to $\mathcal{F}$ when considered as a function of $x$ for every $y \in E$.

(a) If $T \cong M(x, y)$, then $T^{*}$ and $T^{* *}$ exist and $T^{* *}=T . T^{*}$ is a symmetric operator and is the closure of the restriction of $T$ to $\mathcal{F}^{\prime}$; every self-adjoint operator which corresponds to $M(x, y)$ is an extension of $T^{*} . A$ necessary and sufficient condition that $T$ be self-adjoint is that the linear manifold determined by the class $\{M(x, y)+i K(x, y)\}$ is dense in $\mathcal{F}$.

(b) A necessary and sufficient condition that there exists a self-adjoint operator $H \sim M(x, y)$ which is bounded below (above) by the finite number $c$ is that $M(x, y) \gg c K(x, y)(M(x, y) \ll c K(x, y))$. A necessary and sufficient condition that there exists a self-adjoint $H \cong M(x, y)$ with lower bound $\geqq \lambda>-\infty$ and upper bound $\leqq \Lambda<+\infty$ is that $\lambda K(x, y) \ll M(x, y) \ll \Lambda K(x, y)$.

Another result which we must make strong use of in this paper concerns the restriction of a r. k. $K(x, y)$, defined on $E \times E$, to a set $E_{1} \times E_{1} \subseteq E \times E$. The restriction $K_{1}(x, y)$ is a p. m. and its corresponding r. k. s. $\mathscr{f}_{1}$ is obtained from $\mathcal{F}$, the r. k. s. of $K(x, y)$, in the following way. Let $\mathcal{F}_{0}$ be the space of all $g \in \mathcal{F}$ such that $g(x)=0$ for $x \in E_{1}$ and let $\mathcal{F}_{0}^{\perp}$ be its orthogonal complement. Then the space $\mathscr{F}_{1}$ consists of the restrictions of the elements of $\mathcal{F}_{0}^{1}$ to $E_{1}$ with the same norm. The pertinent theorem is as follows.

THEOREM $\mathrm{C}^{(4)}$. If $K$ is the reproducing kernel of the space $\mathcal{F}$ of functions defined on the set $E$ with norm \|\| , then $K$ restricted to the subset $E_{1} \times E_{1} \subseteq E \times E$ is the reproducing kernel of the class $\mathcal{F}_{1}$ of all restrictions of functions of $\mathcal{F}$ to the subset $E_{1}$. For any such restriction, $f_{1} \in \mathcal{F}_{1}$, the norm $\left\|f_{1}\right\|_{1}$ is the minimum of $\|f\|$ for all $f \in \mathcal{F}$ whose restrictions to $E_{1}$ is $f_{1}$.

More detailed information on the topics discussed above will be found in N. Aronszajn $[1 ; 2]$ and A. Devinatz $[1]$.

3. Positive definite functions on columns. Before we prove the results of this section we shall say a few words about the notation which will be used here and in the future. In general the notation will be the same as in IRI.

Vectors in an $n$-dimensional Euclidean space $\left({ }^{5}\right), E_{n}$, will usually be de-

(3) A. Devinatz [1], N. Aronszajn [2].

(4) N. Aronszajn [2].

(5) For convenience we shall also include vectors which have components at $\pm \infty$. 
noted by lower case latin letters and their components by the same letters with superscripts (e. g. $\left.x=\left(x^{(1)}, \cdots, x^{(n)}\right)\right)$. In case we use these letters as scalars it will be clear from the context that we are doing so or else we shall explicitly state this fact. The letters $u_{k}$ shall designate the "unit" vectors, i.e. those vectors for which $u_{k}^{(j)}=\delta_{j k}$, where $\delta_{j k}$ is the Kronecker symbol. We shall write $x \leqq y$ if each component of $x$ is less than or equal to each component of $y$.

We shall also have occasion to use a certain linear subspace of the $(m+n)$ dimensional unitary space. Let $x$ be any vector in $E_{m+n}$ whose last $n$ components are zero and $y$ any vector in $E_{m+n}$ whose first $m$ components are zero. The vectors $z=x+i y, i=(-1)^{1 / 2}$, form a linear subspace of the $(m+n)$ dimensional unitary space. We shall designate this subspace by $Z_{m+n}$. Using the terminology of the complex number field, if $z \in Z_{m+n}$, we shall write $\bar{z}=x-i y, R(z)=x, J(z)=y$.

If $t \in E_{n}$, when we write $\int_{a}^{b} f(t) d \alpha(t)$ we shall mean that $d \alpha(t)$ is a nonnegative measure on $E_{n}$ and that the integral is a Lebesgue-Radon-Stieltjes integral over the closed interval $a \leqq t \leqq b$.

In the following lemmas we shall be working in the 1-dimensional case. These lemmas are proved in much the same manner as the corresponding lemmas in IRI. However, there do exist points here which require some discussion.

Lemma 1(6). Let $f(n)$ be defined for $n=0,1, \cdots, 2 m+1$. Necessary and sufficient conditions that there exists a bounded non-negative measure $d \alpha(t)$ such that

$$
f(n)=\int_{0}^{\infty} t^{n} d \alpha(t), \quad \text { for } 0 \leqq n \leqq\left\{\begin{array}{l}
2 m \quad \text { if } \operatorname{det}|f(j+k)|_{j, k=0}^{m}=0 \\
2 m+1 \text { if } \operatorname{det}|f(j+k)|_{j, k=0}^{m} \neq 0
\end{array}\right.
$$

are:

$$
\begin{array}{rlrl}
f(j+k) & \gg 0, & i, k=0,1, \cdots, m . \\
f(j+k+1) \gg 0, & j, k=0,1, \cdots, m .
\end{array}
$$

Proof. The necessity of these conditions follows immediately. For the sufficiency we have two cases to consider. First of all suppose that the determinant

$$
|f(j+k)|_{j, k=0}^{m}=0 .
$$

The r. k. s. $\mathcal{F}$ associated with $f(j+k)$ is then generated by the elements $\{f(j+k)\}_{k=0}^{m-1}$. Let $f_{1}(j+k)$ be the restriction of $f(j+k)$ to $j, k=0,1, \cdots$, $m-1$, and $\mathcal{F}_{1}$ its corresponding r. k. s. The space $\mathcal{F}$ may be written as $\mathcal{f}$ $=\mathcal{F}_{0} \oplus \mathcal{F}_{0}^{\perp}$, where $\mathcal{F}_{0}$ is the class of all elements in $\mathcal{F}$ which are zero for $j$

(6) This lemma slightly sharpens a theorem of S. Verblunsky [1]. 
$=0,1, \cdots, m-1$, and $\mathcal{F}_{0}^{\perp}$ is its orthogonal complement. The space $\mathcal{F}_{0}^{\perp}$ is generated by the set of elements $\{f(j+k)\}_{k=0}^{m-1}$; but since these elements generate the whole space, $\mathcal{F}_{0}$ can contain only the zero element.

Let $M(j, k)=f(j+k+1)$ be defined for $j, k=0,1, \cdots, m-1$. Then, for fixed $k, M(j, k) \in \mathcal{F}_{1}$. Since $\mathcal{F}_{1}$ is finite-dimensional, by Theorem B the operator

$$
T \cong M(j, k)
$$

must be bounded and therefore self-adjoint. Further by condition (2) it must be positive. For any $g_{1}(j) \in \mathcal{F}_{1}$ we have

$$
T g_{1}(j)=g(j+1)
$$

where $g(j) \in \mathcal{F}$ and $g_{1}$ is its restriction. Let $\left\{E_{t}\right\}$ be the canonical resolution of the identity associated with $T$. Then if $f_{0}(j)=f_{1}(j+0)$ and $r+s=n$, we have

$$
f(n)=(f(j+r), f(j+s))=\left(T^{r} f_{0}, T^{s} f_{0}\right)_{1}=\int_{0}^{\infty} t^{n} d\left(E_{t} f_{0}, f_{0}\right)_{1} .
$$

Suppose now that

$$
|f(j+k)|_{j, k=0}^{m} \neq 0 .
$$

Then it is clearly possible to define a number $f(2 m+2)$ such that

$$
|f(j+k)|_{j, k=0}^{m+1}=0 \text {. }
$$

We can then proceed as before.

Lemma 2. Let $f(x)$ be a continuous function defined on the interval $0 \leqq x \leqq a$. Further, let $f(x+y) \gg 0$ for $0 \leqq x, y \leqq a / 2$. Then there exists an analytic function $F(z)$, defined in the strip $0<R(z)<a$ such that $F(z+w \bar{w}) \gg 0$ for $0<R(z), R(w)$ $<a / 2$ and $F(x)=f(x)$ for $0<x<a$.

With the help of Lemma 1, this lemma may be proved by the same method as Lemma 2 of IRI.

Lemma 3. Let $f(x)$ be as in Lemma 2 and 7 the corresponding r. $k$. s. Let $0<s<a / 8$ and let $I_{s}$ be the set of all $x$ in $[0, a / 2]$ such that $x+4 s \in[0, a / 2]$. Further, let $f_{1}(x+y)$ be the restriction of $f(x+y)$ to $I_{s} \times I_{s}$ and $\mathcal{F}_{1}$ the corresponding r. k. s. If $M_{x_{0}}(x, y)$ is defined on $I_{s} \times I_{s}$ such that for $0 \leqq x_{0} \leqq s, M_{x_{0}}(x, y)$ $=f\left(x+y+x_{0}\right)$, then the operators $T_{x_{0}} \cong M_{x_{0}}(x, y)$ are self-adjoint.

Proof. The space $\mathcal{F}$ may be written as the direct sum $\mathcal{F}=\mathcal{F}_{0} \oplus \mathcal{F}_{0}^{\perp}$, where $\mathcal{F}_{0}$ is the space of elements in $\mathcal{F}$ which are zero on $I_{s}$ and $\mathcal{F}_{0}^{\perp}$ is its orthogonal complement. If $\mathcal{F}_{2}$ is the r. k. s. associated with the $F(z+\bar{w})$ of Lemma 2, then, for every $g \in \mathcal{F}_{0}$, there exists a $g_{2} \in \mathcal{F}_{2}$ whose restriction to the open interval $(0, a / 2)$ coincides with $g(x)$ on this open interval. Therefore, since $g_{2}$ is zero on an interval of the $x$-axis and is analytic, it is identically zero. Since $g(x)$ 
is continuous on $[0, a / 2]$ it must be identically zero on the closed interval. Therefore $\mathcal{F}_{0}$ consists only of the zero element and $\mathcal{F}_{1}$ consists of the restrictions of the elements of $\mathcal{F}$ to $I_{s}$ with the same norm. Consequently, $\mathcal{D}_{T_{x_{0}}}$ is the set of all $g_{1} \in \mathcal{F}_{1}$ such that if $g \in \mathcal{F}$ restricted to $I_{s}$ is $g_{1}$, then $g\left(x+x_{0}\right) \in \mathcal{F}_{1}$ for $x$ in $I_{8}$.

If $T_{x_{0}}$ is not self-adjoint, then since $T_{x_{0}}^{*} \subset T_{x_{0}}$ and $T_{x_{0}}^{* *}=T_{x_{0}}, T_{x_{0}}^{*}$ is not selfadjoint and there exists a nonzero $g_{1} \in \mathcal{F}_{1}$ such that $T_{x_{0}} g_{1}=i g_{1}$, i.e. $g\left(x+x_{0}\right)$ $=i g(x)$ for $x \in I_{8}$, where $g \in \mathcal{F}$ and $g_{1}$ is the restriction to $I_{8}$ of $g$. Now, there exists a $g_{2} \in \mathcal{F}_{2}$ such that $g(x)$ and $g_{2}(x)$ coincide on $(0, a / 2)$. Since $g_{2}(z)$ is analytic, $g\left(x+x_{0}\right)=i g(x)$ for all $x$ for which $x$ and $x+x_{0}$ are in $(0, a / 2)$. By continuity this must also be true for all $x+x_{0}$ which lie in the closed interval $[0, a / 2]$. Consequently, if $x \in I_{s}, x+2 x_{0} \in[0, a / 2]$ and $g\left(x+2 x_{0}\right)=i g\left(x+x_{0}\right)$ $=i^{2} g(x)$. Repeating this argument twice more we get $g\left(x+4 x_{0}\right)=g(x)$ for $x$ in $I_{s}$. Since $g_{2}(z)$ is analytic, for $R(z)+4 x_{0} \in(0, a / 2)$ we have $g_{2}\left(z+4 x_{0}\right)$ $=g_{2}(z)$ and therefore $g_{2}(z)$ may be extended analytically over the whole complex plane. Now,

$$
\left|g_{2}(z)\right|=\left|\left(g_{2}(w), F(w+\bar{z})\right)_{2}\right| \leqq\left\|g_{2}\right\|_{2}(f(2 x))^{1 / 2},
$$

where $x=R(z)$ and $(,)_{2}$ is the inner product of $\mathcal{F}_{2}$. Therefore $g_{2}(z)$ is bounded in every strip $x_{1} \leqq R(z) \leqq x_{2}$ and consequently by Liouville's theorem it must be a constant, which of course must be zero. This contradicts the hypothesis that $g_{1} \neq 0$, which means that $T_{x_{0}}$ is self-adjoint.

Lemma 4. Let $f(x)$ be as in the previous lemmas. Then there exists a spectral measure $d E_{t}$ defined on $\mathcal{F}_{1}$, such that

$$
f(x)=\int_{0}^{\infty} t^{x} d\left(E_{t} f_{0}, f_{0}\right)_{1}\left({ }^{7}\right),
$$

where $f_{0}(x)=f_{1}(x+0)$ and $(,)_{1}$ is the inner product of $\mathcal{F}_{1}$. The measure $d \alpha(t)$ $=d\left(E_{t} f_{0}, f_{0}\right)_{1}$ is uniquely determined. The operators $T_{x_{0}}$ for $0 \leqq x_{0} \leqq s$ are given by

$$
T_{x_{0}}=\int_{0}^{\infty} t^{x_{0}} d E_{t} .
$$

Proof. There exists a spectral measure $d E_{t}$ such that the measure of the interval $[-\infty, 0)$ is zero and

$$
T_{s}=\int_{0}^{\infty} t^{s} d E_{t}
$$

Since every positive self-adjoint operator has a unique positive self-adjoint square root we have, by Lemma 3 ,

$$
T_{s / 2^{n}}=\int_{0}^{\infty} t^{s / 2 n} d E_{t}, \quad n=0,1,2, \cdots .
$$

(7) Cf. S. Bernstein [1] and D. V. Widder [2, 3]. 
Suppose now that $m$ is an integer and $m s / 2^{n} \leqq a / 2$. Then from the fact that the space $\mathcal{F}_{0}$ of Lemma 3 consists of the zero element only, we have

$$
T_{s / 2^{n}}^{m} f_{0}(x)=f\left(x+m s / 2^{n}\right)
$$

and

$$
f\left((p+q) s / 2^{n}\right)=\left(f\left(x+p s / 2^{n}\right), f\left(x+q s / 2^{n}\right)\right)=\left(T_{s / 2^{n}}^{p} f_{0}(x), T_{s / 2^{n}}^{a} f_{0}(x)\right)_{1} .
$$

Therefore,

$$
f\left((p+q) s / 2^{n}\right)=\int_{0}^{\infty} t^{(p+o) s / 2 n} d\left(E_{t} f_{0}, f_{0}\right)_{1}
$$

If we now pass to the limit we get our result. The uniqueness of $d \alpha(t)$ may be proved by the same method as used in Lemma 1 of IRI.

The integral representation of the operators $T_{x_{0}}$ may be obtained by the same method as used in Lemma 4 of IRI. However, since we shall have occasion to use this representation later we shall reproduce the proof here. Consider the operators given by

$$
H_{x_{0}}=\int_{0}^{\infty} t^{x_{0}} d E_{t}
$$

The operators $H_{m s / 2^{n}}$ certainly coincide with $T_{m s / 2^{n}}$, provided $0 \leqq m s / 2^{n} \leqq s$. Since $f_{y}(x)=f_{1}(x+y) \in \mathcal{D}_{T_{m s / 2}}{ }^{n}, f_{y} \in D_{H_{x_{0}}}$ for every $x_{0}$ such that $0 \leqq x_{0} \leqq s$. Therefore

$$
f\left(x+y+m s / 2^{n}\right)=\left(T_{m s / 2^{n}} f_{x}, f_{y}\right)_{1}=\int_{0}^{\infty} t^{m s / 2 n} d\left(E_{t} f_{x}, f_{y}\right)_{1} .
$$

If we choose a sequence of the $m s / 2^{n}$ which approaches any $0<x_{0} \leqq s$ we get

$$
f\left(x+y+x_{0}\right)=\int_{0}^{\infty} t^{x_{0}} d\left(E_{t} f_{x}, f_{y}\right)_{1}=\left(H_{x_{0}} f_{x}, f_{y}\right)_{1} .
$$

Therefore, $H_{x_{0}} f_{y}(x)=f\left(x+y+x_{0}\right)$, which means $H_{x_{0}} \sim M_{x_{0}}(x, y)=f\left(x+y+x_{0}\right)$. Consequently, $H_{x_{0}} \subseteq T_{x_{0}}$ and since both of these operators are self-adjoint we must have $H_{x_{0}}=T_{x_{0} \text {. }}$.

With the help of these lemmas it is now possible to prove a modification of Theorem 5 of IRI. Let $P$ be a set of points $z$ in $Z_{m+n}$ such that $R(z)$ runs over an interval (finite or infinite) in $E_{m}$ which contains the origin and $-\infty$ $<J(z)<\infty$. Let $P / 2$ be the set $z$ in $P$ such that $2 z \in P, 0<s^{(k)}<1 / 8$ sup $\left|z \cdot u_{k}\right|$, where the sup is taken over $z \in P, s=\left(s^{(1)}, \cdots, s^{(m)}, 0, \cdots, 0\right)$, and $P_{s}$ the set of points in $P / 2$ such that $z+4 s \in P / 2$. Further let $a=\left(a^{(1)}, \cdots\right.$, $\left.a^{(m)},-c^{(m+1)}, \cdots,-c^{(m+n)}\right), b=\left(b^{(1)}, \cdots, b^{(m)}, c^{(m+1)}, \cdots, c^{(m+n)}\right)$, and $s_{r}=\left(0, \cdots, 0, s^{(m+1)}, \cdots, s^{(m+n)}\right)$. The numbers $a^{(k)}$ may take on the value 
$-\infty, b^{(k)}$ and $c^{(k)}$ the value $+\infty, a^{(k)} \leqq b^{(k)}$ and $c^{(k)} \geqq 0$.

TheOREM 1. Let $f(z)$ be a continuous complex-valued function defined on $P$. Necessary and sufficient conditions that there exists a bounded non-negative measure $d \alpha(t)$, such that

$$
f(z)=\int_{a}^{b} e^{z \cdot t} d \alpha(t)
$$

are:

(1) $f(z+\bar{w}) \gg 0$ for $z$, w in $P / 2$.

(2) There exists a vector $s>0$ such that for $k=1, \cdots, m$, and $z$, w in $P_{s}$, $\exp \left(s^{(k)} a^{(k)}\right) f(z+\bar{w}) \ll f\left(z+\bar{w}+s^{(k)} u_{k}\right) \ll \exp \left(s^{(k)} b^{(k)}\right) f\left(z+w^{2}\right)\left({ }^{8}\right)$.

(3) There exists a sequence $s_{r} \rightarrow 0$ such that $s_{r}^{(k)}=0$ if $c^{(k)}=\infty, s_{r}^{(k)} \neq 0, c^{(k)}$ $<\infty$, and such that for $k=m+1, \cdots, m+n$ and $r=0,1, \cdots$,

$$
f\left(z+\bar{w}-i s_{r}^{(k)} u_{k}\right)+f\left(z+\bar{w}+i s_{r}^{(k)} u_{k}\right) \gg 2 \cos \left(s_{r}^{(k)} d^{(k)}\right) f(z+\bar{w})\left(^{9}\right) .
$$

Proof. The necessity of these conditions follows immediately by a direct computation. For the sufficiency let $f_{1}(z+\bar{w})$ be the restriction of $f(z+\bar{w})$ to $P_{s} \times P_{s}$ and $\mathcal{F}_{1}$ and $\mathcal{F}$ their corresponding r. k. s. As in Lemma 3 we may easily check that the elements of $\mathcal{F}_{1}$ are the restrictions to $P_{s}$ of the elements of $\mathcal{F}$ with the same norm. Using this fact, if $M(z, w)$ and $N(z, w)$ are defined on $P_{s} \times P_{s}$ such that $M(z, w)=f\left(z+\bar{w}+x^{(k)} u_{k}\right)$ and $N(z, w)=f\left(z+\bar{w}-i y^{(k)} u_{k}\right)$, if we set up the operators

$$
T_{x^{(k)} u k} \cong M(z, w)
$$

and

$$
U_{y^{(k)} u k} \cong N(z, w),
$$

we can proceed exactly as in the proof of Theorem 5 of IRI, using the previous lemmas.

4. Positive definite functions on convex sets. Let $Q$ be an open convex set in the complex plane $\left({ }^{10}\right)$ which contains the origin and which is symmetric with respect to the real axis. Let $Q / 2$ be the set of all $z$ in $Q$ for which $2 z \in Q$. Further, let $d^{(k)}=\sup \left|z \cdot u_{k}\right|$, where the sup is taken over $z \in Q$, $0<s^{(k)}<d^{(k)} / 8, s=s^{(1)}+i s^{(2)}$, and $Q_{s}$ the set of all $z$ in $Q / 2$ such that $z \pm 4 s \in Q / 2$ and $z \pm 4 \bar{s} \in Q / 2$. It is obvious that $Q / 2$ and $Q_{s}$ are convex sets in the complex plane which are open, contain the origin, and are symmetric with re-

$\left.{ }^{8}\right)$ In case $a^{(k)}=-\infty$, the corresponding left "inequality" is clearly redundant. In case $b^{(k)}=+\infty$, let us agree that the right "inequality" is also redundant.

(9) Define $0 \cdot \infty=0$.

(10) In case $Q$ reduces to a segment of the real or imaginary axis we shall mean that $Q$ is open with respect to the topology of these straight lines. 
spect to the real axis. Let $f(z)$ be a continuous complex-valued function defined on $Q$ such that $f(z+w \bar{w}) \gg 0$ for $z$ and $w$ in $Q / 2$. Let $f_{1}(z+\bar{w})$ be the restriction of $f(z+\bar{w})$ to $Q_{s} \times Q_{s}$. Suppose further that $\mathcal{F}$ is the r. k. s. associated with $f(z+\bar{w})$ and $\mathcal{F}_{1}$ the r. k. s. associated with $f_{1}(z+v \bar{w})$ with inner product $(,)_{1}$. If $\mathcal{F}_{0}$ is the set of elements in $\mathcal{F}$ which are zero on $Q_{s}$ and $\mathcal{F}_{0}^{\perp}$ the orthogonal complement of $\mathcal{F}_{0}$, then we know by Theorem $C$ that $\mathcal{F}_{1}$ is the restriction of the elements of $\mathcal{F}_{0}^{\perp}$ to $Q_{s}$ with the same norm. We know further that all elements of the form $f(z+w \bar{w})$ for $w \in Q_{s}$ belong to $\mathcal{F}_{0}^{\perp}$.

Let us consider the elements $f\left(z+\bar{w}_{0}+x\right)$, for $x$ real, $w_{0} \in Q_{\varepsilon}$, and $w_{0}+x$ $\in Q / 2$. We may then write

$$
f\left(z+\bar{w}_{0}+x\right)=K_{0}^{\perp}\left(z, w_{0}+x\right)+K_{0}\left(z, w_{0}+x\right),
$$

where $K_{0}^{\perp}\left(z, w_{0}+x\right) \in \mathcal{F}_{0}^{\perp}$ and $K_{0}\left(z, w_{0}+x\right) \in \mathcal{F}_{0}$. Let us now consider the complex numbers $w(x)$, where $\Im(w(x))=\Im\left(w_{0}\right)$ and $R(w(x))$ varies so that $w(x) \in Q / 2$. Since $Q_{s}$ is open these complex numbers actually trace out some line segment in $Q_{s}$ which we shall designate by $L_{s}$ and an extension in $Q / 2$ which we shall designate by $L$. Let $L^{\prime}$ be the projection of $L$ on the real axis. If we consider the restriction of $f(z+\bar{w})$ to $L \times L$, then this is exactly the same p. m. which we get if we consider the restriction of $f(z+\bar{w})$ to $L^{\prime} \times L^{\prime}$. Therefore this restriction is an analytic function of $x$ (see Lemma 2) and all functions which belong to the r. k. s. of this restriction are analytic. Now, for any $z \in Q / 2$ and $w(x) \in L_{s}, K_{0}(z, w(x))=0$. Therefore, since for any fixed $z \in Q / 2, \bar{K}_{0}(w(x), z)$ when considered as a function of $x$ belongs to the r. k. s. corresponding to the restriction of $f(z+\bar{w})$ to $L \times L$, and since it is zero on $L_{s}$, it must be zero for all of $L$. Consequently, for $w_{0} \in Q_{s}$ and $z \in Q / 2$,

$$
f\left(z+\bar{w}_{0}+x\right)=K_{0}^{\perp}\left(z, w_{0}+x\right),
$$

which means that $f\left(z+\bar{w}_{0}+x\right) \in \mathcal{F}_{0}^{\perp}$.

On the space $\mathcal{F}_{1}$ set up the operators $T_{x} \cong M_{x}(z, w)=f(z+\bar{w}+x)$ for $|x| \leqq s^{(1)}$. The domain of $T_{x}$ consists of those elements $g_{1} \in \mathcal{F}_{1}$ such that $g(z+x) \in \mathcal{F}_{1}$, where $g \in \mathcal{F}_{0}^{\perp}$ and $g_{1}$ is the restriction of $g$ to $Q_{8}$. These operators are self-adjoint (see Lemma 3 and IRI Lemma 6 ). Further, since $f\left(z+\bar{w}_{0}+x\right)$ $\in \mathcal{F}_{0}^{\perp}$ for $w_{0} \in Q_{8}$ and any $x$ such that $w_{0}+x \in Q / 2$, then for any integer $n$ such that $w_{0}+n x \in Q / 2$,

$$
T_{x}^{n} f_{1}\left(z+\bar{w}_{0}\right)=f\left(z+\bar{w}_{0}+n x\right) .
$$

This means that if $g_{1} \in D_{T_{x}}$, then $T_{x}^{n} g_{1}\left(w_{0}\right)=g\left(w_{0}+n x\right)$, where $g \in \mathcal{F}_{0}^{\perp}$ and $g_{1}$ is the restriction of $g$. This follows from the fact that

$$
\begin{aligned}
& T_{x g_{1}}^{n}\left(w_{0}\right)=\left(T_{x}^{n} g_{1}(z), f_{1}\left(z+\bar{w}_{0}\right)\right)_{1}=\left(g_{1}(z), T_{x}^{n} f_{1}\left(z+\bar{w}_{0}\right)\right)_{1} \\
& =\left(g(z), f\left(z+\bar{w}_{0}+n x\right)\right) \text {. }
\end{aligned}
$$


Let us also consider the operators

$$
V_{\nu} \cong N_{y}(z, w)=f(z+\bar{w}-i y)
$$

for $|y| \leqq s^{(2)}$. If $h(z)=\sum_{k=1}^{n} \xi_{k} f_{1}\left(z+\bar{w}_{k}\right)$ with $\bar{w}_{k}+i y \in Q_{s}$, then clearly

$$
\left\|V_{y} h\right\|_{1}=\|h\|_{1} \text {. }
$$

In general, for any $g \in \mathcal{F}_{1}$,

$$
\left\|V_{y} g\right\|_{1} \leqq\|g\|_{1} .
$$

Consequently, $V_{y}$ is a bounded operator and $V_{y} g_{1}(z)=g(z+i y)$, where $g \in \mathcal{F}_{0}^{\perp}$ and $g_{1}$ is the restriction of $g$ to $Q_{8}$. From this remark it is easy to see that if each of the points $\bar{w}+i y_{1}$ and $\bar{w}+i y_{2}$ belong to $Q_{s}$ then

$$
V_{y_{1}} V_{y_{2}} f_{1}(z+\bar{w})=V_{y_{1}+y_{2}} f_{1}(z+\bar{w})=V_{y_{2}} V_{y_{1}} f_{1}(z+\bar{w}) .
$$

Further, it is clear that $V_{y} g$ is a strongly continuous function of $y$ for every $g \in \mathcal{F}_{1}, V_{0}=I$, and since $V_{y^{*}} \cong \bar{N}_{y}(w, z)=\bar{f}(\bar{z}+w-i y)=f(z+\bar{w}+i y)$ (Aronszajn $\left[2\right.$, p. 372]), we have $V_{y}^{*}=V_{-y}$.

We shall now show that each of the operators $V_{y}$ permutes with each of the operators $T_{x}$. We have first of all $T_{x} f_{1}(z+\bar{w})=f(z+\bar{w}+x)$. Since, for $z \in Q / 2$, $f(z+\bar{w}+x) \in \mathcal{F}_{0}^{\perp}$, we have $V_{y} T_{x} f_{1}(z+\bar{w})=f(z+\bar{w}+x+i y)$. On the other hand, $V_{y} f_{1}(z+\bar{w})=f(z+\bar{w}+i y)$. Now the element $f(z+\bar{w}+i y)$ for $z \in Q_{s}$ is the restriction to $Q_{s}$ of the function $K_{0}^{\perp}(z, w-i y)$ which was defined above. Therefore, $T_{x} V_{y} f_{1}(z+\bar{w})=K_{0}^{\perp}(z+x, w-i y)$. However, by (4.1) we see that $K_{0}^{\perp}(z+x, w-i y)=f(z+\bar{w}+x+i y)$ for $z, w$ in $Q_{s}$. This means that $T_{x}$ and $V_{y}$ permute on the set $\mathcal{F}_{1}^{\prime}$. Now Theorem B tells us that $T_{x}$ is the closure of its restriction to $\mathcal{F}_{1}^{\prime}$. Therefore $V_{y}$ permutes with $T_{x}$.

Let us now discuss the question of the extension of a complex-valued positive linear functional on a space of complex-valued functions whose field of scalars is the complex number field. The result which we state below is, we believe, well known and has been used implicitly in the literature. However, we have never seen a formal statement of this result so we think it is well, for the sake of completeness, to give it here.

Lemma 5(11). Let $X$ be a complex linear space of complex-valued functions $x(t)$, which are defined over some domain $D$. Let $S$ be a subspace of $X$ and $l(x) a$ linear functional on $S$ with range in the complex number field. Further, let us suppose that $l(x)$ is real whenever $x(t)$ is real on some fixed subdomain $D_{1} \subseteq D$, $l(x) \geqq 0$ whenever $x(t) \geqq 0$ on $D_{1}$, and for every $x_{0} \in X$ which is real on $D_{1}, \sup l(x)$ $<\infty$ and inf $l(x)>-\infty$, where the sup is taken over all $x \in S$ which are real on $D_{1}$ and $x(t) \leqq x_{0}(t)$ on $D_{1}$ and the inf is taken over all $x \in S$ which are real on $D_{1}$ and such that $x(t) \geqq x_{0}(t)$ on $D_{1}$. Then there exists a linear functional defined on all of $X$ which is an extension of $l(x)$ and which retains all of the properties of $l$.

(11) I am indebted to Professor Harry Pollard for an enlightening discussion of this lemma. 
Proof. Let $X^{\prime}$ be the set of all real elements in $X$ and $S^{\prime}=S \cap X^{\prime}$. Then $X^{\prime}$ is a real linear space and $S^{\prime}$ is a linear subspace of $X^{\prime}$. By hypothesis the restriction $l^{\prime}$, of $l$ to $S^{\prime}$, is a real positive linear functional. Then, by the extension theorem for real positive linear functionals (see Achieser and Krein [1]), $l^{\prime}$ may be extended to a positive functional $L^{\prime}$ over $X^{\prime}$. Now, it is necessary to extend $L^{\prime}$ to the complex linear manifold determined by $S$ and $X^{\prime}$ so that $L^{\prime}$ coincides with $l$ on $S$. But this is easily done. We may write every element in this manifold in the form $y=x+a x^{\prime}$, where $x \in S, x^{\prime} \in X^{\prime}$, and $a$ is a complex number. If $y_{1}$ and $y_{2}$ are any such elements let us consider $c_{1} y_{1}+c_{2} y_{2}$ $=c_{1} x_{1}+c_{2} x_{2}+c_{1} a_{1} x_{1}^{\prime}+c_{2} a_{2} x_{2}^{\prime}$, where the coefficients are complex numbers. Let us then write

$$
L\left(c_{1} y_{1}+c_{2} y_{2}\right)=c_{1} l\left(x_{1}\right)+c_{2} l\left(x_{2}\right)+c_{1} a_{1} L^{\prime}\left(x_{1}{ }^{\prime}\right)+c_{2} a_{2} L^{\prime}\left(x_{2}{ }^{\prime}\right) .
$$

This is a linear functional which coincides with $l$ on $S$ and $L^{\prime}$ on $X^{\prime}$. Now, extend $L$ to all of $X$ in any way so as to be linear and the lemma is proved.

It should be pointed out that the condition that $l$ be real for real elements of $S$ is independent of the condition of positivity and is needed. For, there exist spaces in which the failure of this condition is enough to insure that there can be no positive extension. Consider, for example, $S$ as the complex linear space which consists of polynomial functions $P(t),-\infty<t<\infty$, with complex coefficients and such that $P(1)=0$. Define $l(P)=P(0)+i P^{\prime}(1)$, where $i=(-1)^{1 / 2}$ and the prime stands for the derivative. If $P(t) \geqq 0$, then $P^{\prime}(1)=0$ and $l(P) \geqq 0$. However, $l$ is not necessarily real for real $P(t)$. Suppose now that $P(t)$ is a real polynomial such that $l(P)$ is not real, i.e. $P^{\prime}(1) \neq 0$. Write $P(t)=P_{+}(t)-P_{-}(t)$, where $P_{+}(t)=\max [P(t), 0]$ and $P_{-}(t)=-\min [P(t), 0]$. Let $X$ be the complex linear space generated by the space $S$ and the functions $P_{+}$and $P_{-}$. Suppose it would be possible to extend $l$ to $L$ defined on $X$ such that $L$ is a positive linear functional. Then $L(P)=l(P)=L\left(P_{+}\right)-L\left(P_{-}\right)$. But since $P_{+}$and $P_{-}$are both positive, $l(P)$ is real which gives a contradiction.

With all of these preliminaries out of the way we may now state and prove the theorem we want.

Theorem 2. Let $f(z)$ be a continuous complex-valued function defined on $Q$. Necessary and sufficient conditions that there exists a bounded non-negative measure $d \alpha\left(t_{1}, t_{2}\right)$ which is zero on the complement of the interval $a \leqq t_{1} \leqq b$, $-c \leqq t_{2} \leqq c$ ( $a$ may take the value $-\infty, b$ and $c$ the value $\left.+\infty\right)$ and such that

$$
f(z)=\int_{-c}^{c} \int_{a}^{b} e^{x t_{1}+i y t_{2}} d \alpha\left(t_{1}, t_{2}\right)
$$

are:

(1) $f(z+\bar{w}) \gg 0$ for $z, w \in Q / 2$.

(2) There exists a positive sequence $r_{n} \rightarrow 0$ such that for $n=1,2, \cdots$,

$$
e^{r_{n} a} f(z+\bar{w}) \ll f\left(z+\bar{w}+r_{n}\right) \ll e^{r_{n} b} f(z+\bar{w}),
$$


for all $z$ and $w$ in $Q / 2$ for which the functions are defined $\left({ }^{12}\right)$.

(3) There exists a sequence $s_{n} \rightarrow 0$ such that for $n=1,2, \cdots, s_{n}=0$ if $c=\infty, s_{n} \neq 0$ if $c<\infty$, and

$$
f\left(z+\bar{w}-i s_{n}\right)+f\left(z+\bar{w}+i s_{n}\right) \gg 2 \cos \left(s_{n} c\right) f(z+\bar{w})\left({ }^{9}\right),
$$

for all $z$ and $w$ in $Q / 2$ for which the functions are defined.

Before we proceed with the proof of this theorem let us notice certain things. If the set $Q$ reduces to one half of the real axis and if $a=-\infty$, $b=0$, this is equivalent to the Hausdorff [1]-S. Bernstein [1]-Widder [1] theorem on completely monotone functions. For $a=-\infty, b=+\infty$, this is the $\mathrm{S}$. Bernstein $[1]$-Widder $[2 ; 3]$ theorem on exponentially convex functions. If the set $Q$ reduces to an interval on the imaginary axis and if $c=\infty$, this is a theorem of M. Krein [1] and if $Q$ is the whole imaginary axis this is the well known theorem of S. Bochner. Finally, for $a=-\infty, b=c=+\infty$, this is the correct version of an incorrect theorem stated by M. Livshitz [1, Theorem 3].

According to Livshitz, condition (1) of our Theorem 2 is necessary and sufficient that there exist a bounded non-negative measure $d \alpha(t)$, defined on the real line such that

$$
f(z)=\int_{-\infty}^{\infty} e^{z t} d \alpha(t)
$$

That condition (1) of Theorem 2 is not sufficient for such a representation can easily be seen by the following simple counter example. Let $d \alpha_{1}(t)$ be a bounded non-negative measure such that the integral

$$
f(x)=\int_{-\infty}^{\infty} e^{x t} d \alpha_{1}(t)
$$

exists for $x \geqq 0$. Suppose further that the measure is normed so that $f(0)=1$. Let $d \alpha_{2}(t)$ be a non-negative measure different from $d \alpha_{1}(t)$ and such that $\int_{-\infty}^{\infty} d \alpha_{2}(t)=1$. Form the function

$$
g(y)=\int_{-\infty}^{\infty} e^{i y t} d \alpha_{2}(t)
$$

for $-\infty<y<\infty$. Then if $d \alpha_{1}\left(t_{1}\right) \alpha_{2}\left(t_{2}\right)$ represents the product measure, the function

$$
F(z)=f(x) g(y)=\int_{-\infty}^{\infty} \int_{-\infty}^{\infty} e^{x t_{1}+i y t_{2}} d \alpha_{1}\left(t_{1}\right) \alpha_{2}\left(t_{2}\right)
$$

(12) For some types of domains, for example an interval, the existence of only one number, $r$, for which the "inequalities" hold is sufficient. We have been unable to determine whether this is true for all convex sets. As before, if $b=+\infty$, consider the corresponding "inequality" as redundant. 
satisfies all of the conditions of the theorem of Livshitz. Therefore if his theorem were true, there exists a bounded non-negative measure $d \alpha_{3}(t)$ such that

$$
F(z)=\int_{-\infty}^{\infty} e^{z t} d \alpha_{3}(t) .
$$

Now, if we take $\Im(z)=0$, we get

$$
F(x)=f(x)=\int_{-\infty}^{\infty} e^{x t} d \alpha_{3}(t),
$$

from which it follows that $d \alpha_{1}=d \alpha_{3}$. On the other hand if $R(z)=0$,

$$
F(i y)=g(y)=\int_{-\infty}^{\infty} e^{i y t} d \alpha_{3}(t),
$$

from which it follows that $d \alpha_{2}=d \alpha_{3}$. This gives a contradiction. The theorem of Livshitz will become true if we allow $f(z)$ to be analytic instead of merely continuous.

Proof of Theorem 2. The necessity follows immediately by a direct computation. For the sufficiency let us consider a set $Q_{s}$ and the set of operators $\left\{T_{x}\right\}$ which we have constructed previously. By the same method as used in Lemma 4 we may show the existence of a spectral measure $d G_{t}$ such that for $0 \leqq x \leqq s^{(1)}$,

$$
T_{x}=\int_{0}^{\infty} t^{x} d G_{t} .
$$

However, in this case, for any $x$ such that $|x| \leqq s^{(1)}, T_{-x}$ is the inverse of $T_{x}$ and therefore the origin has a zero measure with respect to $d G_{t}$. Therefore, there exists a spectral measure $d E_{t}$ such that for $|x| \leqq s^{(1)}$,

$$
T_{x}=\int_{-\infty}^{\infty} e^{x t} d E_{t} .
$$

From the remarks made at the beginning of this section, for any $x$ such that $z+x \in Q_{s}$ and for any $g \in \mathcal{D}_{T_{x}}$, where $T_{x}$ is defined by the above integral if $|x|>s^{(1)}$, we have $T_{x} g(z)=g(z+x)$. This follows from the fact that for any $x_{1}$ and integer $n$ such that $\left|x_{1}\right| \leqq s^{(1)}$ and $n x_{1}=x, T_{x_{1}}^{n}=T_{x}$.

There exists a countable set of mutually orthogonal subspaces, $\left\{\mathcal{X}_{k}\right\}_{k=1}^{\infty}$, whose direct sum is $\mathcal{F}_{1}$, which reduces every $T_{x}$, and the restriction of $T_{x}$ to $\mathcal{X}_{k}$ is a bounded self-adjoint operator which we shall denote by $T_{x}^{(k)}$. Therefore, we may write

$$
T_{x}^{(k)}=\int_{a_{k}}^{b_{k}} e^{x t} d E_{t}^{(k)}
$$


where $a_{k}$ and $b_{k}$ are finite and $d E_{t}^{(k)}$ is a spectral measure which is zero outside of the interval $\left[a_{k}, b_{k}\right]$. If $f_{k}(z, w)$ is the r. k. of $\mathcal{X}_{k}$, then if $w+x \in Q_{s}$,

$$
\begin{aligned}
T_{x}^{(k)} f_{k}(z, w) & =\left(T_{x}^{(k)} f_{k}(u, w), f_{k}(u, z)\right)_{1}=\left(f_{k}(u, w), T_{x}^{(k)} f_{k}(u, z)\right)_{1} \\
& =T_{x}^{(k)} f_{k}(w, z)=\overline{f_{k}}(w+x, z)=f_{k}(z, w+x) .
\end{aligned}
$$

Let

$$
A_{k}=\int_{a_{k}}^{b_{k}} t d E_{t}^{(k)}
$$

It is clear that if

$$
A_{\delta}^{(k)}=\frac{T_{\delta}^{(k)}-I}{\delta},
$$

then for any $g \in \mathcal{X}_{k}$ we have

$$
A_{\delta}^{(k)} g \rightarrow A_{k} g,
$$

where the bold arrow indicates convergence in the strong topology of $\mathcal{F}_{1}$. However, if $\delta$ is sufficiently small,

$$
A_{\delta}^{(k)} g(z)=\frac{g(z+\delta)-g(z)}{\delta} .
$$

Therefore, if $x \in R(z)$, since $\mathcal{F}_{1}$ is a r. k. s. we get

$$
A_{k} g(z)=\frac{\partial g(z)}{\partial x} \text {. }
$$

In $\mathscr{X}_{k}$ let $\mathscr{D}_{B_{k^{\prime}}{ }^{\prime}}$ be the set of all $g(z) \in \mathscr{X}_{k}$ such that for $y=\mathfrak{J}(z), \partial g(z) / \partial y$ exists and belongs to $\mathscr{X}_{k}$. We shall then define

$$
B_{k}^{\prime} g(z)=\frac{\partial g(z)}{\partial y} .
$$

The first thing that we wish to show is that $\mathscr{D}_{B_{k}{ }^{\prime}}$ is dense in $\mathscr{X}_{k}$. To this end let us consider the operators

$$
W_{\delta}=\frac{V_{\delta}-I}{\delta}, \quad|\delta| \leqq s^{(2)}\left(V_{\delta} \text { defined at beginning of } \S 4\right) .
$$

If $P_{k}$ is the projection operator whose range is $\mathscr{X}_{k}$, then since $W_{\delta}$ permutes with every $T_{x}$ it also permutes with $P_{k}$. Consequently, if $W$ is the strong limit of $W_{\delta}$ as $\delta \rightarrow 0$, then $W$ also permutes with $P_{k}$ and therefore $\mathcal{X}_{k}$ reduces $W$. Now, clearly if $g \in \mathcal{D}_{W}$, then 


$$
W g=\frac{\partial g}{\partial y} .
$$

Therefore, if we can show that $\mathcal{D}_{W}$ is dense in $\mathcal{F}_{1}$, we shall have shown that $\mathcal{D}_{B_{k}}{ }^{\prime}$ is dense in $\mathscr{X}_{k}$.

Let us consider an element of the form

$$
g(z)=\sum_{k=1}^{n} \xi_{k} f_{1}\left(z+\bar{w}_{k}\right) .
$$

Since $Q_{s}$ is open there exists a real $r$ such that $w_{k}-2 i r \in Q_{s}$ for $k=1, \cdots, n$. If we set up the element

$$
g_{r}(z)=\frac{1}{r} \int_{0}^{r} V_{t} g(z) d t,
$$

then $g_{r} \in \mathcal{F}_{1}$ and $g_{r} \rightarrow g$ as $r \rightarrow 0$. Therefore, this class of elements of the form $g_{r}$ is dense in $\mathcal{F}_{1}$. Further, if $|\delta| \leqq|r|$, using (4.2) we get

$$
W_{\delta} g_{r}=\frac{1}{\delta r}\left[\int_{r}^{r+\delta} V_{t} g d t-\int_{0}^{\delta} V_{t} g d t\right] \rightarrow W_{r} g
$$

as $\delta \rightarrow 0\left({ }^{13}\right)$. Consequently $\mathscr{D}_{W}$ is dense in $\mathcal{F}_{1}$.

Let us now show that for $g \in \mathscr{D}_{W^{*}}$,

$$
W^{*} g=-\frac{\partial g}{\partial y} .
$$

To this end let us consider the elements

$$
f_{r}(z)=\frac{1}{r} \int_{0}^{r} V_{t} f_{1}(z+\bar{w}) d t,
$$

where $w$ is fixed and $r$ is taken so small that $\bar{w}+i r \in Q_{s}$. Then since $W f_{r}(z)$ $=W_{r} f_{1}(z+\bar{w})$ we have

$$
\begin{aligned}
\left(g(z), W f_{r}(z)\right)_{1}=\left(g(z), W_{r} f_{1}(z+\bar{w})\right)_{1} & =\left(g(z), \frac{f_{1}(z+\bar{w}+i r)-f_{1}(z+\bar{w})}{r}\right)_{1} \\
& =\left(W^{*} g(z), f_{r}(z)\right)_{1} .
\end{aligned}
$$

Therefore, since $f_{r}(z) \rightarrow f_{1}(z+\bar{w})$, we get

$$
W^{*} g(w)=\lim _{r \rightarrow 0}\left(W^{*} g, f_{r}\right)_{1}=\lim _{r \rightarrow 0} \frac{g(w-i r)-g(w)}{r}=-\frac{\partial g(w)}{\partial y} .
$$

This shows that if $W_{k}^{*}$ is the restriction of $W^{*}$ to $\mathcal{X}_{k}$, then $W_{k}^{*} \subseteq-B_{k}^{\prime}$.

(18) We have followed here a technique of N. Dunford and I. E. Segal [1]. 
If we consider the operator

$$
B_{k}=-i B_{k}^{\prime}
$$

then we have shown that

$$
-i W_{k} \subseteq B_{k} \text { and }\left(-i W_{k}\right)^{*} \subseteq B_{k} .
$$

Therefore

$$
B_{k}^{*} \subseteq\left(-i W_{k}\right)^{*} \subseteq B_{k} .
$$

Further, since it is clear that $B_{k}$ is a closed operator we have that $\mathscr{D}_{B_{k}}{ }^{*}$ is dense in $\mathscr{X}_{k}$ and therefore $B_{k}^{*}$ is a closed symmetric operator with $B_{k}^{* *}=B_{k}$.

We wish to show now that the deficiency index of $B_{k}^{*}$ is $(m, m)$ and therefore it can be extended to a self-adjoint operator. Let us first show that if $h(z) \in \mathcal{F}_{1}$, then $\bar{h}(\bar{z}) \in \mathcal{F}_{1}$ and

$$
(\bar{h}(\bar{z}), \bar{g}(\bar{z}))_{1}=\overline{(h(z), g(z))_{1}} .
$$

Consider first any $h \in \mathcal{F}_{1}^{\prime}$, i.e.

$$
h(z)=\sum_{k=1}^{n} \xi_{k} f_{1}\left(z+\bar{w}_{k}\right) .
$$

Then

$$
\bar{h}(\bar{z})=\sum_{k=1}^{n} \bar{\xi}_{k} f_{1}\left(z+w_{k}\right) \in \mathcal{F}_{1}^{\prime}
$$

It is clear that if $g$ is also in $\mathcal{F}_{1}^{\prime}$, then

$$
(\bar{h}(\bar{z}), \bar{g}(\bar{z}))_{1}=\overline{(h(z), g(z))_{1}} .
$$

Suppose now that $h(z)$ is any element of $\mathcal{F}_{1}$. Then there exists a sequence $\left\{h_{n}\right\}_{n=1} \subseteq \mathcal{F}_{1}^{\prime}$ such that $h_{n} \rightarrow h$. Therefore $\left\|h_{n}(z)-h_{m}(z)\right\|_{1}=\left\|\bar{h}_{n}(\bar{z})-\bar{h}_{m}(\bar{z})\right\|_{1} \rightarrow 0$ as $n, m \rightarrow \infty$. This means there exists a $k(z) \in \mathcal{F}_{1}$ such that $\bar{h}_{n}(\bar{z}) \rightarrow k(z)$. But since strong convergence in $\mathcal{F}_{1}$ implies pointwise convergence in $\mathcal{F}_{1}$, we must have $k(z)=\bar{h}(\bar{z})$. Clearly if $\bar{g}(\bar{z}) \in \mathcal{F}_{1}$, then the inner product of $\bar{h}(\bar{z})$ and $\bar{g}(\bar{z})$ has the desired relation.

Let $J$ be the conjugation operator defined by the formula

$$
J g=\bar{g}(\bar{z}) .
$$

It is clear that $J$ permutes with each of the operators $T_{x}$ for $|x| \leqq s^{(1)}$. Therefore $\mathscr{X}_{k}$ reduces $J$. If $J_{k}$ is the restriction of $J$ to $\mathscr{X}_{k}$, it is also clear that $J_{k}$ permutes with $B_{k}$ and therefore with $B_{k}^{*}$. This means that $B_{k}^{*}$ is real with respect to the conjugation $J_{k}$ and therefore its deficiency index is of the form $(m, m)$. 
If $S_{k}$ is the Cayley transform of $B_{k}^{*}$, then the deficiency spaces of $B_{k}^{*}$ are given by $\mathfrak{F C}_{k}^{+}=\mathcal{X}_{k} \ominus \mathcal{D}_{S_{k}}$ and $\mathfrak{F C}_{k}^{-}=\mathcal{X}_{k} \ominus \mathcal{R}_{S_{k}}$, where $\mathcal{D}_{S_{k}}$ and $\mathcal{R}_{S_{k}}$ are respectively the domain and range of $S_{k}$. The spaces $\mathcal{F C}_{k}^{+}$and $\mathcal{F C}_{k}^{-}$are given respectively by the elements in $\mathscr{X}_{k}$ which satisfy the equations

$$
B_{k} g=i g \text { and } B_{k} h=-i h .
$$

The solutions of these partial differential equations yield

$$
g(z)=e^{-y} g(x) \text { and } h(z)=e^{y} h(x) .
$$

Furthermore, any functions of this form which belong to $\mathcal{X}_{k}$, for $x+i y \in Q_{s}$, are in $\mathfrak{H C}_{k}^{+}$and $\mathfrak{H}_{k}^{-}$respectively.

We wish to show now that if $g(z) \in \mathcal{F C}_{k}^{+}$then $\bar{g}(z) \in \mathcal{F C}_{k}^{+}$and $\|g\|_{1}=\|\bar{g}\|_{1}$. It will follow by a similar argument that if $h(z) \in \mathfrak{F C}_{\boldsymbol{k}}^{-}$, then $\bar{h}(z) \in \mathfrak{F}_{\boldsymbol{k}}^{-}$. Let $K(z, w)$ be the r. k. of $\mathfrak{H}_{\boldsymbol{k}}^{+}$and $\left\{\phi_{n}(z)\right\}$ a complete orthonormal system in $\mathfrak{H C}_{k}^{+}$. If $w=v+i v$, then since $\phi_{n}(z)=e^{-v} \phi_{n}(x), K(z, w)$ has the expansion

$$
K(z, w)=\sum_{n} e^{-y} \phi_{n}(x) e^{-v} \Phi_{n}(u)=e^{-(y+v)} K(x, u) .
$$

Now, $\mathfrak{F C}_{k}^{+}$reduces the operator $A_{k}$ and therefore each of the operators $T_{x}^{(k)}$. In order to see this we notice first that since $A_{k}$ and $B_{k}$ are differential operators, $\mathcal{D}_{A_{k}}=\mathscr{X}_{k}$ and every element of $\mathcal{X}_{k}$ is continuous in $R(z)$ and $\mathfrak{J}(z)$, it follows that $A_{k}$ permutes with $B_{k}$. Further, since $A_{k}$ is self-adjoint this means that $A_{k}$ permutes with $B_{k}^{*}$ and therefore $A_{k} S_{k} \subseteq S_{k} A_{k}$. This means that $A_{k}$ takes $\mathcal{D}_{S_{k}}$ into itself. On the other hand since every element of $\mathfrak{F C}_{k}^{+}$is the form $e^{-y} g(x)$ and every element of this form which belongs to $\mathcal{X K}_{k}$ is in $\mathfrak{F C}_{k}^{+}$, it follows that $A_{k}$ takes $\mathfrak{H C}_{k}^{+}$into itself. Therefore $A_{k}$ may be written as the direct sum of two operators defined respectively on $\mathcal{D}_{S_{k}}$ and $\mathfrak{F}_{k}^{+}$. This means that if $P_{k}^{+}$is the projection operator whose domain is $\mathcal{H}_{k}$ and range is $\mathfrak{F}_{\boldsymbol{k}}^{+}$, then $A_{k}$ and therefore $T_{x}^{(k)}$ permutes with $P_{k}^{+}$.

The kernel $K(z, w)$ is given by the formula

$$
K(z, w)=P_{k}^{+} f_{k}(z, w),
$$

where, as we have noted before, $f_{k}(z, w)$ is the r. k. of $\mathcal{X}_{k}$. Therefore,

$$
\begin{aligned}
K(z, w) & =P_{k}^{+} T_{u}^{(k)} f_{k}(x+i y, i v)=T_{u}^{(k)} P_{k}^{+} f_{k}(x+i y, i v) \\
& =T_{x}^{(k)} T_{u}^{(k)} P_{k}^{+} f_{k}(i y, i v)=T_{x+u}^{(k)} P_{k}^{+} f_{k}(i y, i v) .
\end{aligned}
$$

Therefore, $K(x, u)$ is a function of $x+u$ which means that

$$
K(z, w)=K^{\prime}(z+w)=e^{-(y+v)} K^{\prime}(x+u) .
$$

This means that $K(z, w)$ is real from which our assertion follows (see $\mathrm{N}$. Aronszajn [2, p. 354]).

Define a linear operator $S_{k}^{\prime}$, with $\mathcal{D}_{\mathcal{S}_{k^{\prime}}}=\mathfrak{F C}_{k}^{+}$and $\mathcal{R}_{S_{k^{\prime}}}=\mathfrak{F C}_{\boldsymbol{k}}^{-}$, by the formula 


$$
S_{k}^{\prime} e^{-y} g(x)=e^{i \theta} e^{y} g(x),
$$

where $\theta$ is any real number. The function $e^{y} g(x) \in \mathcal{H C}_{\mathbf{k}}^{-}$since $J e^{-y} g(x)=e^{y} \bar{g}(x)$ $\in \mathcal{F C}_{\mathbf{k}}^{-}$. Further, since

$$
\left\|e^{-y} g(x)\right\|_{1}=\left\|J e^{-y} g(x)\right\|_{1}=\left\|e^{y} g(x)\right\|_{1},
$$

it follows that $S_{k}^{\prime}$ is an isometric operator. Consequently, $U_{k}=S_{k} \oplus S_{k}^{\prime}$ is a unitary operator and this gives rise to a self-adjoint extension of $B_{k}^{*}$ whose Cayley transform is $U_{k}$. Clearly if $C_{k}$ is any self-adjoint extension of $B_{k}^{*}$, then $C_{k} \subseteq B_{k}$. If the deficiency index of $B_{k}^{*}$ is not $(0,0)$ there exists an infinite number of self-adjoint extensions of $B_{k}^{*}$ of the type which we have described.

Suppose that $C_{k}$ is a self-adjoint extension of $B_{k}^{*}$ of the type we have described. In order that $A_{k}$ permutes with $C_{k}$, it is sufficient that $A_{k}$ permutes with $U_{k}$. However, we have already shown that $A_{k} S_{k} \subseteq S_{k} A_{k}$ and since clearly $A_{k} S_{k}^{\prime} \subseteq S_{k}^{\prime} A_{k}$, we have $A_{k} U_{k}=U_{k} A_{k}$. Therefore every $T_{x}^{(k)}$ permutes with $C_{k}$ and consequently if $C$ is the direct sum of the operators $\left\{C_{k}\right\}_{k=1}^{\infty}$, then each operator $T_{x}$ permutes with the self-adjoint operator $C$.

If condition (3) of Theorem 2 is fulfilled with $c<\infty$, we shall show that $C$ has the bound $c$. Since $s_{n} \rightarrow 0$, we can find an $N$ such that for $n>N$ and for all $w \in Q_{s}, w \pm i s_{n} \in Q / 2$. By Theorem $\mathrm{B}$, condition (3) means that

$$
\frac{1}{2}\left[V_{s_{n}}+V_{s_{n}}^{*}\right] \geqq \cos \left(s_{n} c\right) I .
$$

If $s_{n}$ is chosen sufficiently small, then the right-hand side is positive. Consequently we get

$$
\frac{1}{4}\left[V_{s_{n}}+V_{s_{n}}^{*}\right]^{2} \geqq \cos ^{2}\left(s_{n} c\right) I .
$$

Therefore,

$$
I-\frac{1}{4}\left[V_{s_{n}}+V_{s_{n}}^{*}\right]^{2} \leqq \sin ^{2}\left(s_{n} c\right) I .
$$

Now, from the fact that $\left\|V_{s_{n}}\right\| \leqq 1$ it follows that $V_{s_{n}} V_{s_{n}}^{*} \leqq I$ and $V_{s_{n}}^{*} V_{s_{n}} \leqq I$ and from this it follows immediately that

$$
\frac{i^{2}}{4}\left[V_{s_{n}}-V_{s_{n}}^{*}\right]^{2} \leqq I-\frac{1}{4}\left[V_{s_{n}}+V_{s_{n}}^{*}\right]^{2} \leqq \sin ^{2}\left(s_{n} c\right) I .
$$

Since $i / 2\left[V_{s_{n}}-V_{s_{n}}^{*}\right]$ is self-adjoint it follows that

$$
-\sin \left(s_{n} c\right) I \leqq \frac{i}{2}\left[V_{s_{n}}-V_{s_{n}}^{*}\right] \leqq \sin \left(s_{n} c\right) I .
$$

From this we get 


$$
-\frac{\sin \left(s_{n} c\right) I}{s_{n}} \leqq \frac{i}{2}\left[\frac{V_{s_{n}}-I}{s_{n}}+\frac{V_{-s_{n}}-I}{-s_{n}}\right] \leqq \frac{\sin \left(s_{n} c\right) I}{s_{n}} .
$$

From this inequality it immediately follows that

$$
-c I \leqq-i W \leqq c I,
$$

which means that $-i W$ is self-adjoint and coincides with $C$.

Let $\left\{F_{t}\right\}$ be the canonical resolution of the identity associated with the operator $C$. Consider the group of unitary operators

$$
U_{\nu}=\int_{-\infty}^{\infty} e^{i y t} d F_{t}
$$

Let $g \in \mathcal{F}_{1}$ for which

$$
U_{y} g=\int_{-d}^{d} e^{i y t} d F_{t} g,
$$

where $d>0$ is a finite number. The set of such elements is dense in $\mathcal{F}_{1}$ and it may easily be verified that

$$
U_{y} g=\sum_{n=0}^{\infty} \frac{(i y)^{n}}{n !} C^{n} g,
$$

where the convergence is taken in the strong sense.

Let us now consider the set of points $x_{0}+i y \in Q_{s}$ for which $x_{0}$ is fixed. Consider the function

$$
G_{x_{0}}(\zeta)=\int_{-d}^{d} e^{x t} d F_{t} g\left(x_{0}+i y\right),
$$

where $\zeta=x+i y$. The function $G_{x_{0}}(\zeta)$ coincides with $g\left(x_{0}+i y\right)$ for $x=0$. Further,

$$
\frac{\partial G_{x_{0}}(\zeta)}{\partial x}=\int_{-d}^{d} t e^{x t} d F_{t} g\left(x_{0}+i y\right)
$$

and

$$
\begin{aligned}
\frac{\partial G_{x_{0}}(\zeta)}{\partial y} & =i \int_{-d}^{d} e^{x t} d C F_{t} g\left(x_{0}+i y\right) \\
& =i \int_{-d}^{d} t e^{x t} d F_{t} g\left(x_{0}+i y\right) .
\end{aligned}
$$

This last formula for the partial derivative with respect to $y$ follows immediately if we first integrate by parts, then take the partial derivative, and then integrate by parts again. This shows that $G_{x_{0}}(\zeta)$ is an analytic function of $\zeta$. 
Therefore if $z \pm i y \in Q_{s}$ we have

$$
U_{y} g(z)=\sum_{n=0}^{\infty} \frac{\partial^{n} g(z)}{\partial y^{n}} \frac{y^{n}}{n !}=g(z+i y) .
$$

This means that since $U_{y}$ is bounded and $\mathcal{F}_{1}$ is a r. k. s., for any $h \in \mathcal{F}_{1}, U_{y} h(z)$ $=h(z+i y)$, provided $z \pm i y \in Q_{s}$. Therefore if $i y \in Q_{s}$ and $f_{z}(w)=f_{1}(w+\bar{z})$, then

$$
U_{y} f_{0}(z)=\left(U_{y} f_{0}(w), f_{z}(w)\right)_{1}=\overline{U_{-y} f_{z}}(0)=\overline{f_{z}}(-i y)=f_{1}(z+i y) .
$$

Let us consider now the set $2 Q_{s}$, i.e. the set of all $z \in Q$ such that $z / 2 \in Q_{s}$. Suppose that $z \in 2 Q_{s}$ and $x=R(z), y=J(z)$. Let $x_{1}=x / 2, y_{1}=y / 2$. There exists an $x_{2}$ and an integer $n$ such that $\left|x_{2}\right| \leqq s^{(1)}$ and $n x_{2}=x_{1}$. If $T_{x_{1}}=T_{x_{2}}^{n}$, then by condition (2) of Theorem 2, Theorem $\mathrm{C}$ and our previous remarks, we have

$$
f(z)=\left(T_{x_{1}} U_{y_{1}} f_{0}, T_{x_{1}} U_{-y} f_{0}\right)_{1}=\int_{a}^{b} \int_{-c}^{c} e^{x t_{1}+i y t_{2}} d\left(E_{t_{1}} F_{t_{2}} f_{0}, f_{0}\right)_{1},
$$

where, as we have noted before, $\left\{E_{t_{1}}\right\}$ is the resolution of the identity associated with the set $\left\{T_{x}\right\}$ and $\left\{F_{t_{2}}\right\}$ is the canonical resolution of the identity of the operator $C$. Since $E_{t_{1}}$ permutes with $F_{t_{2}},\left\{E_{t_{1}} F_{t_{2}}\right\}$ is a resolution of the identity which of course gives rise to a spectral measure $d E_{t_{1}} F_{t_{2}}$.

Let us now consider the linear space $S$ of functions of the form

$$
g\left(t_{1}, t_{2}\right)=\sum_{k=1}^{n} \xi_{k} e^{x t_{1}+i y_{k} t_{2}}
$$

where $x_{k}=z_{k}+i y_{k} \in Q$ and $a \leqq t_{1} \leqq b,-c \leqq t_{2} \leqq c$. On $S$ set up the linear functional

$$
l(g)=\sum_{k=1}^{n} \xi_{k} f\left(z_{k}\right) .
$$

By the integral representation of $f(z)$ given above it is clear that if $g\left(t_{1}, t_{2}\right)$ is a function of the form (4.3) with $z_{k} \in 2 Q_{s}$, then $g\left(t_{1}, t_{2}\right) \geqq 0$ implies $l(g) \geqq 0$. However, if we allow $s=s^{(1)}+i s^{(2)} \rightarrow 0$, then the set $2 Q_{s}$ approaches the set $Q$ and therefore $l(g)$ is a positive linear functional on all of $S$.

Let us extend $S$ to the linear space $X$, which consists of functions of the form (4.3) where now the $y_{k}$ vary between $-\infty$ and $\infty$ and $x_{k}$ is in $Q$. The positive linear functional satisfies all of the conditions of Lemma 5 and therefore may be extended to a positive linear functional $L$, defined on all of $X$. If we set

$$
F(z)=L\left(e^{x t_{1}+i y t_{2}}\right),
$$

it is clear that $F(z)$ coincides with $f(z)$ on $Q$. Further, $F(z+\bar{w}) \gg 0$ for $R(z)$, $R(w)$ in $Q / 2$. 
It remains to prove that $F(z)$ is a continuous function of $z$. First of all we know that for $z$ in $Q, F(z)(=f(z))$ is continuous. Let $\mathcal{F}_{2}$ be the r. k. s. associated with $F(z+\bar{w})$. Then if $x \in Q$ and $\delta_{1}$ and $\delta_{2}$ are sufficiently small,

$$
\begin{aligned}
\mid F(x+ & \left.i y+\delta_{1}+i \delta_{2}\right)-F(x+i y) \mid \\
& =\left|\left(F(w+x / 2), F\left(w+x / 2-i y+\delta_{1}-i \delta_{2}\right)-F(w+x / 2-i y)\right)_{2}\right| \\
& \leqq\|F(w+x / 2)\|_{2}\left\|F\left(w+x / 2-i y+\delta_{1}-i \delta_{2}\right)-F(w+x / 2-i y)\right\|_{2} \\
& =(f(x))^{1 / 2}\left(f\left(x+2 \delta_{1}\right)+f(x)-2 R\left(f\left(x+\delta_{1}+i \delta_{2}\right)\right)\right)^{1 / 2} .
\end{aligned}
$$

As $\delta_{1}+i \delta_{2} \rightarrow 0$, the right-hand side goes to zero which proves our result.

Now, by the definition of the linear functional $L$, if $g\left(t_{1}, t_{2}\right) \geqq 0$ for $a \leqq t_{1} \leqq b$ and $-c \leqq t_{2} \leqq c$, then $L(g) \geqq 0$. Consequently, since for an $r$ suitably small

$$
\begin{aligned}
& \sum_{j, k=1}^{n} \xi_{j} \bar{\xi}_{k}\left[F\left(z_{k}+\bar{z}_{j}+r\right)-e^{r a} F\left(z_{k}+\bar{z}_{j}\right)\right] \\
&=L\left(\left(e^{r t_{1}}-e^{r a}\right)\left|\sum_{k=1}^{n} \xi_{k} e^{x t_{1}+i y k t_{2}}\right|^{2}\right),
\end{aligned}
$$

if $t_{1} \geqq a$, the right side and therefore the left side is positive. Similarly, one shows that

$$
F(z+\bar{w}+r) \ll e^{r b} F(z+v \bar{w})
$$

and that there exists a sequence $s_{n} \rightarrow 0$ such that

$$
F\left(z+\bar{w}-i s_{r}\right)+F\left(z+\bar{w}+i s_{n}\right) \gg 2 \cos \left(s_{n} c\right) F(z+\bar{w}) .
$$

Therefore, by Theorem 1 there exists a spectral measure $d E_{t_{1}, t_{2}}$ such that

$$
F(z)=\int_{-c}^{c} \int_{a}^{b} e^{x t_{1}+i y t_{2}} d\left(E_{t_{1}, t_{2}} F_{0}, F_{0}\right) .
$$

This concludes the proof of our theorem.

Using the notation of $\$ 2$, suppose that $Q^{\prime}$ is an open convex set in $Z_{m+n}$ which contains the origin and with the further conditions that $R(z)$ fills out an interval in $E_{m}$ as $z$ runs over $Q^{\prime}$ and $z \in Q^{\prime}$ implies $z-2 i y^{(k)} u_{k} \in Q^{\prime}$ for $k=m+1, \cdots, m+n$. The set $Q^{\prime}$ is then symmetric in each component of $\Im(z) . Q^{\prime} / 2$ shall be the set of all $z$ in $Q^{\prime}$ such that $2 z \in Q^{\prime}$. If we let $a=\left(a^{(1)}, \cdots, a^{(m)},-c^{(m+1)}, \cdots,-c^{(m+n)}\right), b=\left(b^{(1)}, \cdots, b^{(m)}, c^{(m+1)}, \cdots\right.$, $\left.c^{(m+n)}\right), t=\left(t^{(1)}, \cdots, t^{(m+n)}\right), s_{r}=\left(s_{r}^{(1)}, \cdots, s_{r}^{(m+n)}\right)$ where $a^{(k)} \leqq b^{(k)}, a^{(k)}$ may take on the value $-\infty, b^{(k)}$ the value $+\infty$, and $c^{(k)} \geqq 0$ and is finite, then we have the following theorem.

THEOREM 3. Let $f(z)$ be a continuous complex-valued function defined on $Q^{\prime}$. Necessary and sufficient conditions that there exists a bounded non-negative measure $d \alpha(t)$ such that 


$$
f(z)=\int_{a}^{b} e^{z \cdot t} d \alpha(t)
$$

are:

(1) $f(z+\bar{w}) \gg 0$ for $z, w \in Q^{\prime} / 2$.

There exists a sequence $s_{r} \rightarrow 0$ such that:

(2) For $k=1, \cdots, m$ and $r=1,2, \cdots, s_{r}^{(k)}>0$ and

$$
\exp \left(s_{r}^{(k)} a^{(k)}\right) f(z+\bar{w}) \ll f\left(z+\bar{w}+s_{r}^{(k)} u_{k}\right) \ll \exp \left(s_{r}^{(k)} b^{(k)}\right) f(z+\bar{w})
$$

for all $z$ and $w$ in $Q^{\prime} / 2$ for which the functions are defined.

(3) For $k=m+1, \cdots, m+n$ and $r=1,2, \cdots, s_{r}^{(k)} \neq 0$ and

$$
f\left(z+\bar{w}-i s_{r}^{(k)} u_{k}\right)+f\left(z+\bar{w}+i s_{r}^{(k)} u_{k}\right) \gg 2 \cos \left(s_{r}^{(k)} c^{(k)}\right) f(z+\bar{w})
$$

for all $z$ and w in $Q^{\prime} / 2$ for which the functions are defined.

The necessity of these conditions is immediate. The proof of sufficiency will proceed exactly along the same lines as the proof of Theorem 2. However, since here $c^{(k)}<\infty$, condition (3) tells us that all of the differential operators

$$
C_{u_{k}} g(z)=-i \frac{\partial g(z)}{\partial y^{(k)}}, \quad \quad k=m+1, \cdots, m+n,
$$

are bounded and therefore permute with one another. In the case where these operators are unbounded we have been unable as yet to prove the permutability. However, we think it is very likely that Theorem 3 is true even when some of the $c^{(k)}$ are unbounded.

5. A problem of moments. By making use of methods similar to some of the methods initiated in the last section we can prove the following theorem.

THEOREM 4. Let $\left\{\mu_{m, n}\right\}$ be a sequence of numbers defined for $m$ $=0,1,2, \cdots$ and $n=0, \pm 1, \pm 2, \cdots . A$ necessary and sufficient condition that there exists a non-negative bounded measure $d \alpha\left(t_{1}, t_{2}\right)$ which is zero outside the strip $-\infty<t_{1}<\infty,-\pi \leqq t_{2} \leqq \pi$ and such that

$$
\mu_{m, n}=\int_{-\pi}^{\pi} \int_{-\infty}^{\infty} t_{1}^{m} e^{i n t_{2}} d \alpha\left(t_{1}, t_{2}\right)
$$

is

$$
\mu_{m_{1}+m_{2}, n_{1}-n_{2}} \gg 0 \text {. }
$$

In order that the measure $d \alpha\left(t_{1}, t_{2}\right)$ be uniquely determined it is sufficient that the moment problem corresponding to the sequence $\left\{\mu_{m, 0}\right\}$ be determined (i.e. there exists a unique non-negative measure $d \beta(t)$ such that $\mu_{m, 0}=\int_{-\infty}^{\infty} t^{m} d \beta(t)$ ).

Proof. Let $\mathcal{F}$ be the r. k. s. corresponding to the r. k. $\mu_{m_{1}+m_{2}, n_{1}-n_{2}}$. The operators 


$$
U_{8} \cong \mu_{m_{1}+m_{2}, n_{1}-n_{2}-8}
$$$$
s=0, \pm 1, \cdots,
$$

clearly form a group of unitary operators with $U_{s}^{*}=U_{-8}$. Let us also consider the operator

$$
T \cong \mu_{m_{1}+m_{2}+1, n_{1}-n_{2}} \text {. }
$$

By Theorem B of $\$ 2$ we know that $T^{*}$ and $T^{* *}$ exist and $T^{* *}=T$. Therefore $T^{*}$ is a closed symmetric operator. Further by the same methods as used in $\$ 4$ we see that the operator given by

$$
J g(m, n)=\bar{g}(m,-n)
$$

is a conjugation operator and that $J$ permutes with $T$. Therefore $J$ permutes with $T^{*}$ which means that $T^{*}$ has deficiency index of the form $(m, m)$.

Let $V$ be the Cayley transform of $T^{*}$. The deficiency spaces of $T^{*}$ are given by $\mathcal{H}^{+}=\mathcal{F} \ominus D_{V}$ and $\mathcal{H}^{-}=\mathcal{F} \ominus R_{V}$. $\mathfrak{H}^{+}$and $\mathcal{H}^{-}$consist respectively of elements which satisfy the respective equations

$$
T g=i g \text { and } T h=-i h,
$$

that is to say elements of the form

$$
g(m, n)=i^{m} g(0, n) \text { and } h(m, n)=(-i)^{m} h(0, n) .
$$

Conversely, it is clear that any elements of this form which belong to $\mathcal{F}$ belong respectively to $\mathfrak{H C}^{+}$and $\mathfrak{H C}^{-}$.

Let us now show that if $i^{m} g(0, n) \in \mathcal{H}^{+}$, then $i^{m} \bar{g}(0,-n) \in \mathcal{F}^{+}$and both of these elements have the same norm. It will then follow by a similar argument that if $(-i)^{m} h(0, n) \in \mathfrak{H}^{-}$, then $(-i)^{m} \bar{h}(0,-n) \in \mathfrak{F}^{-}$. First of all let us show that $\mathrm{FC}^{+}$reduces any operator $U_{s}$. It is clear that $T^{*}$ and $U_{s}$ permute on $\mathcal{F}^{\prime}$. Since $T^{*}$ is the closure of its restriction to $\mathcal{F}^{\prime}$ it follows immediately that $U_{8} T^{*} \subseteq T^{*} U_{8}$. From this it follows that $U_{8} V \subseteq V U_{8}$. This means that $U_{8}$ takes $\mathcal{D}_{V}$ into itself. Further, since it is clear that $U_{s}$ takes $\mathcal{H}^{+}$into itself, $U_{s}$ may be written as the direct sum of two operators whose domains are respectively $\mathscr{D}_{V}$ and $\mathfrak{H}^{+}$. Therefore if $P$ is the projection operator whose range is $\mathfrak{H}^{+}$, we have $U_{8} P=P U_{s}$.

Let $K((m, n),(r, s))$ be the r. k. of $\mathcal{H}^{+}$and $\left\{\phi_{k}\right\}$ a complete orthonormal system in $\mathrm{FC}^{+}$. Then

$$
K((m, n),(r, s))=\sum_{k} \phi_{k}(m, n) \Phi_{k}(r, s)=i^{m-r} \sum_{k} \phi_{k}(0, n) \Phi_{k}(0, s) .
$$

On the other hand

$$
\begin{aligned}
K((m, n),(r, s)) & =P \mu_{m+r, n-s}=P U_{-s} \mu_{m+r, n} \\
& =U_{-8} P \mu_{m+r, n}=U_{n} U_{-8} P \mu_{m+r, 0} \\
& =U_{n-s} P \mu_{m+r, 0}
\end{aligned}
$$


This means

$$
K((m, n),(r, s))=i^{m-r} K^{\prime}(n-s),
$$

where $K^{\prime}(n-s)=K((0, n),(0, s))$. Therefore $K$ is the product of two positive matrices. If $\mathfrak{F}_{1}^{+}$is the r. k. s. corresponding to $i^{m-r}$ and $\mathfrak{F C}_{2}^{+}$the r. k. s. corresponding to $K^{\prime}$, then $\mathfrak{F}^{+}$is the direct product of $\mathfrak{F}_{1}^{+}$and $\mathfrak{F}_{2}^{+}$(see $\mathrm{N}$. Aronszajn [2]). The elements of $\mathfrak{F C}_{2}^{+}$are the restriction of the elements of $\mathfrak{F C}^{+}$ to the set consisting of points of the form $(0, n)$. Therefore, if $i^{m} g(0, n) \in \mathfrak{F C}^{+}$, then $g(0, n) \in \mathfrak{F}_{2}^{+}$and $\bar{g}(0,-n) \in \mathfrak{F}_{2}^{+}$with $\|g(0, n)\|_{2}=\|\bar{g}(0,-n)\|_{2}$. Consequently,

$$
\left\|i^{m} g(0, n)\right\|=\left\|i^{m}\right\|_{1}\|g(0, n)\|_{2}=\left\|i^{m}\right\|_{1}\|\bar{g}(0,-n)\|_{2}=\left\|i^{m} \bar{g}(0,-n)\right\| .
$$

Define the linear operator $V^{\prime}$ with domain $\mathfrak{C}^{+}$and range $\mathfrak{F}^{-}$by the formula

$$
V^{\prime} i^{m} g(0, n)=e^{i \theta}(-i)^{m} g(0, n),
$$

where $\theta$ is any real number. It is clear that $V^{\prime}$ is linear. Further, since

$$
\left\|i^{m} g(0, n)\right\|=\left\|J i^{m} g(0,-n)\right\|=\left\|(-i)^{m} \bar{g}(0,-n)\right\|=\left\|(-i)^{m} g(0, n)\right\|,
$$

it is clear that $V^{\prime}$ is an isometric operator and therefore $U=V \oplus V^{\prime}$ is a unitary operator. The operator $U$ gives rise to a self-adjoint extension of $T^{*}$ whose Cayley transform is $U$. Therefore, if the deficiency index is not $(0,0)$ there exist an infinite number of self-adjoint extensions of $T^{*}$ of the type we have described. Clearly, if $H$ is a self-adjoint extension of $T^{*}$, then $H \subseteq T$.

Suppose that $H$ is a self-adjoint extension of $T^{*}$ of the type we have described. Every operator $U_{s}$ permutes with $H$. In order to show this it is sufficient to show that $U_{s}$ permutes with $U$. However, we have already shown that $U_{s}$ permutes with $V$ and since clearly $U_{s}$ permutes with $V^{\prime}$, the result follows.

If $\left\{E_{t_{1}}\right\}$ is the canonical resolution of the identity associated with $H$ and $\left\{F_{t_{2}}\right\}$ the canonical resolution of the identity associated with $U_{1}$, then by the permutability of $U_{1}$ and $H$, it follows that $E_{t_{1}, t_{2}}=E_{t_{1}} F_{t_{2}}$ is a projection and the family $\left\{E_{t_{1}, t_{2}}\right\}$ is a resolution of the identity. Therefore if $f_{0}$ $=\mu_{m+0, n-0}$, we have

$$
\mu_{m, n}=\left(H^{m} U_{n} f_{0}, f_{0}\right)=\int_{-\pi}^{\pi} \int_{-\infty}^{\infty} t_{1}^{m} e^{i n t_{2}} d\left(E_{t_{1}, t_{2}} f_{0}, f_{0}\right) .
$$

This completes the proof of representation.

To prove the statement about the uniqueness of the measure $d \alpha\left(t_{1}, t_{2}\right)$ let us suppose that the moment problem associated with the sequence $\left\{\mu_{m, 0}\right\}$ is determined. Let $\mathcal{F}_{1}$ be the r. k. s. associated with the r. k. $\mu_{m+r, 0}$. The operator 
must be self-adjoint, for otherwise the moment problem associated with $\left\{\mu_{m, 0}\right\}$ cannot have a unique solution. Therefore, the function $i^{m}$ cannot belong to $\mathcal{F}_{1}$. This means the space $\mathfrak{H C}^{+}$consists only of the zero element. For suppose to the contrary that there exists an element $g(m, n)$ in $\mathfrak{F}^{+}$which is not identically zero. Then there exists an $n_{0}$ such that $g\left(0, n_{0}\right) \neq 0$. Now the restrictions of the elements of $\mathcal{F}$ to the points $\left(m, n_{0}\right)$ belong to $\mathcal{F}_{1}$. Therefore $g\left(m, n_{0}\right)$ $=i^{m} g\left(0, n_{0}\right) \in \mathcal{F}_{1}$ which means that $i^{m} \in \mathcal{F}_{1}$. This gives a contradiction. Therefore the operator $T$ must be self-adjoint. By the same method as used in IRI Lemma 1 , the self-adjointness of $T$ implies the uniqueness of $d \alpha\left(t_{1}, t_{2}\right)$.

We take this opportunity to note the following errata in IRI in addition to that published in this journal vol. 74 (1953) p. 536:

p. 64 , line 8 . Omit " $s_{r}^{(k)} d(k)<\pi$."

p. 65 , line -9 . For " $\mathcal{L}_{0}$ " read " $\mathcal{L}_{0}^{\frac{1}{1}}$."

p. 70, line -4 . Omit " $y_{n} d \leqq \pi$."

p. 72, line 4 . For " $x \cdot u_{k}$ " read " $x^{(k)} u_{k}$."

pp. 72, 73. For " $T_{x \cdot u_{j}}$," " $T_{x \cdot u_{k}}$ " and " $U_{y \cdot u_{k}}$ " read resp. " $T_{x}^{(j)} u_{j}$ " " $T_{x}^{(k)} u_{k}$ "

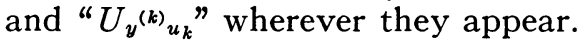

\section{REFERENCES}

N. ACHIESER and M. KREIN

1. On certain problems in the theory of moments (in Russian), Kharkoff, 1938, $254 \mathrm{pp}$.

N. AronszajN

1. La thêorie gênérale des noyaux reproduisants et ses applications, Première Partie, Proc. Cambridge Philos. Soc. vol. 39 (1943) p. 133.

2. The theory of reproducing kernels, Trans. Amer Math. Soc. vol. 68 (1950) pp. 337-404.

S. BERNSTEIN

1. Sur les fonctions absolument monotones, Acta Math. vol. 52 (1929) pp. 1-66.

A. Devinatz

1. Integral representations of positive definite functions, Trans. Amer. Math. Soc. vol. 74 (1953) pp. 56-77.

N. Dunford and I. E. Segal

1. Semi-groups of operators and the Weierstrass theorem, Bull. Amer. Math. Soc. vol. 52 (1946) pp. 911-914.

F. HAUSDORFF

1. Summationsmethoden und Momentfolgen, Math. Zeit. vol. 9 (1921), I, pp. 74-109, II, pp. 280-299.

M. KREIN

1. Sur le problème du prolongement des fonctions hermitiennes positive at continues, C. R. (Doklady) Acad. Sci. URSS. N. S. vol. 26 (1940) pp. 17-22.

M. LivshiTZ

1. On an application of the theory of Hermitian operators to the generalized problem of moments, C. R. (Doklady) Acad. Sci. URSS. N. S. vol. 44 (1944) pp. 3-7.

J. A. Shohat and J. D. TAMARKIN

1. The problem of moments, Mathematical Surveys, vol. 2, American Mathematical Society, 1943, $144 \mathrm{pp}$.

B. v. Sz. NAGY

1. Spectraldarstellung linear Transformationen des Hilbertschen Raumes, Ergibnisse der Mathematik und ihrer Grenzgebiete, no. 5, 1942, 80 pp. 
S. VERBLUNSKY

1. On a problem of moments, Proc. Cambridge Philos. Soc. vol. 45 (1949) pp. 1-4.

D. V. WIDDER

1. Necessary and sufficient conditions for the representation of a function as a Laplace integral, Trans. Amer. Math. Soc. vol. 33 (1931) pp. 851-892.

2. Necessary and sufficient conditions for the representation of a function by a doubly infinite Laplace integral, Bull. Amer. Math. Soc. vol. 40 (1934) pp. 321-326.

3. The Laplace transform, Princeton, 1941, $406 \mathrm{pp}$.

The Illinois Institute of Technology, Chicago, Ill.

The Institute for Advanced Study, Princeton, N. J. 